\title{
MODEL STRUCTURES ON TRIANGULATED CATEGORIES
}

\author{
XIAOYAN YANG \\ Department of Mathematics, \\ Northwest Normal University, Lanzhou, 730070, China \\ e-mail: yangxy@nwnu.edu.cn
}

(Received 3 December 2012; revised 17 August 2013; accepted 29 January 2014; first published online 18 December 2014)

\begin{abstract}
We define model structures on a triangulated category with respect to some proper classes of triangles and give a general study of triangulated model structures. We look at the relationship between these model structures and cotorsion pairs with respect to a proper class of triangles on the triangulated category. In particular, we get Hovey's one-to-one correspondence between triangulated model structures and complete cotorsion pairs with respect to a proper class of triangles. Some applications are given.
\end{abstract}

2000 Mathematics Subject Classification. 18E30, 55U35, 18G25.

1. Introduction. Triangulated categories were introduced by Grothendieck and Verdier in the early sixties as the proper framework for doing homological algebra in an abelian category. Also, such categories were independently introduced in homotopy theory by Puppe in [13]. Since their introduction, they have turned out quite useful in algebraic geometry, stable homotopy theory and representation theory. Examples for this can be found in duality theory by Hartshorne [6] and Iversen [10] or in the fundamental work on perverse sheaves by Bernstein et al. [2].

Let $\mathcal{T}$ be a triangulated category with triangulation $\Delta$. Beligiannis [3] investigated the triangulated category $\mathcal{T}$ using relative homological algebra which parallels the homological algebra in an exact category in the sense of Quillen. To develop homological algebra, a class of triangles $\xi \subseteq \Delta$, called the proper class of triangles, is specified. This class is closed under translations, and it satisfies the analogous formal properties of a proper class of short exact sequences. Moreover, $\xi$-projective objects, $\xi$-projective resolution, $\xi$-projective dimension and their duals are introduced and studied [3, Section 4].

Recall that a model category structure is a way of formally introducing homotopy theory into a category on an abelian category. Model structures are usually not constructed for their own sake but to understand the localization of a given category. The fundamental theorem in this respect is [7, Theorem 1.2.10], which shows that the localization of the category is equivalent to a sub-quotient of it. Although the category is assumed to be bicomplete, the full strength of the assumption is not used. There are many important examples of model category structures on abelian categories. The most famous is the projective model structure on $C(R)$ [7, Section 2.3], the category of unbounded complexes of $R$-modules, where $R$ is a ring. Hovey [8] made a general study of Quillen model structures and gave a method of constructing model structures on abelian categories. Gillespie [5] defined model structures on exact categories and 
got Hovey's one-to-one correspondence between exact model structures and complete cotorsion pairs. But homological algebra itself is encompassed in Quillen's notion of a model category and so there ought to be model structures on triangulated categories with respect to proper classes of triangles describing homological algebra in these categories.

Let $\mathcal{T}=(\mathcal{T}, \Sigma, \Delta)$ be a triangulated category and $\xi \subseteq \Delta$ a proper class of triangles, where $\Sigma$ is the suspension functor and $\Delta$ is the triangulation. In this paper, we introduce triangulated model structures and make a general study of relationship between triangulated model structures and cotorsion pairs with respect to $\xi$. We prove the following main result.

Theorem A. Suppose $\mathcal{T}$ has a triangulated model structure with respect to $\xi$. Let $\mathcal{C}$ denote the full subcategory of cofibrant objects, $\mathcal{F}$ denote the full subcategory of fibrant objects and $\mathcal{W}$ denote the full subcategory of trivial objects. Then,

(1) $\mathcal{W}$ is a thick subcategory of $\mathcal{T}$; and

(2) $(\mathcal{C}, \mathcal{F} \cap \mathcal{W})$ and $(\mathcal{C} \cap \mathcal{W}, \mathcal{F})$ are complete cotorsion pairs with respect to $\xi$.

Conversely, given classes $\mathcal{C}, \mathcal{F}$ and $\mathcal{W}$ satisfying the two conditions above, there is a unique triangulated model structure on $\mathcal{T}$ with respect to $\xi$ such that $\mathcal{W}$ is the class of trivial objects, $\mathcal{C}$ is the class of cofibrant objects and $\mathcal{F}$ is the class of fibrant objects.

This paper is organized as follows: In Section 1, we give some notions and draw some basic consequences of the proper class $\xi$. In Sections 2 and 3, we define and conduct a brief study of triangulated model structures with respect to $\xi$. These are triangulated categories with a model structure that is compatible with $\xi$ and is entirely analogous to Hovey's definition of an abelian model category, which appeared in [8]. We also define cotorsion pairs with respect to $\xi$ in $\mathcal{T}$, and get Hovey's one-toone correspondence between triangulated model structures and cotorsion pairs with respect to $\xi$. Finally, some applications are given in Section 4.

2. Some basic facts in triangulated categories. This section is devoted to discussing the axioms of a proper class of triangles and drawing some basic consequences for use throughout this paper. The basic reference for triangulated categories is the monograph of Neeman [12]. For terminology we shall follow [3] and [11].

Triangulated categories. Let $\mathcal{T}$ be an additive category, and $\Sigma: \mathcal{T} \rightarrow \mathcal{T}$ be an additive functor. Let $\operatorname{Diag}(\mathcal{T}, \Sigma)$ denote the category whose objects are diagrams in $\mathcal{T}$ of the form $X \stackrel{\mu}{\rightarrow} Y \stackrel{v}{\rightarrow} Z \stackrel{\omega}{\rightarrow} \Sigma X$, and morphisms between two objects $X_{i} \stackrel{\mu_{i}}{\rightarrow}$ $Y_{i} \stackrel{\nu_{i}}{\rightarrow} Z_{i} \stackrel{\omega_{i}}{\rightarrow} \Sigma X_{i}, i=1,2$, are triples of morphisms $f: X_{1} \rightarrow X_{2}, g: Y_{1} \rightarrow Y_{2}$ and $h: Z_{1} \rightarrow Z_{2}$, such that the following diagram commutes:

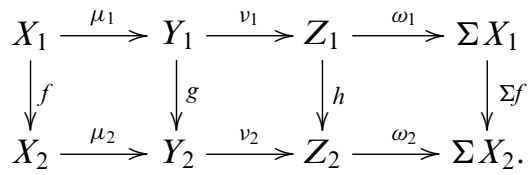

Such a morphism is said to be an isomorphism if $f, g, h$ are isomorphisms in $\mathcal{T}$.

A triple $(\mathcal{T}, \Sigma, \Delta)$ is called a triangulated category, where $\mathcal{T}$ is an additive category, $\Sigma$ is an autoequivalence of $\mathcal{T}$ and $\Delta$ is a full subcategory of $\operatorname{Diag}(\mathcal{T}, \Sigma)$ which satisfies the following axioms. The elements of $\Delta$ are then called triangles. 
(TR1) Every diagram isomorphic to a triangle is a triangle. For every object $X$ in $\mathcal{T}$, the diagram $X \stackrel{1}{\rightarrow} X \rightarrow 0 \rightarrow \Sigma X$ is a triangle. Every morphism $\mu: X \rightarrow Y$ in $\mathcal{T}$ can be embedded into a triangle $X \stackrel{\mu}{\rightarrow} Y \rightarrow Z \rightarrow \Sigma X$.

(TR2) $X \stackrel{\mu}{\rightarrow} Y \stackrel{\nu}{\rightarrow} Z \stackrel{\omega}{\rightarrow} \Sigma X$ is a triangle if and only if $Y \stackrel{\nu}{\rightarrow} Z \stackrel{\omega}{\rightarrow} \Sigma X \stackrel{-\Sigma \mu}{\rightarrow} \Sigma Y$ is so.

(TR3) Given triangles $X \stackrel{\mu}{\rightarrow} Y \stackrel{\nu}{\rightarrow} Z \stackrel{\omega}{\rightarrow} \Sigma X$ and $X^{\prime} \stackrel{\mu^{\prime}}{\rightarrow} Y^{\prime} \stackrel{\nu^{\prime}}{\rightarrow} Z^{\prime} \stackrel{\omega^{\prime}}{\rightarrow} \Sigma X^{\prime}$, then each commutative diagram



can be completed to a morphism of triangles (but not necessarily uniquely).

(TR4) The octahedral axiom. For the formulation of this we refer to Proposition 2.1.

Proposition 2.1 [3, 2.1]. Let $\mathcal{T}$ be an additive category equipped with an autoequivalence $\Sigma: \mathcal{T} \rightarrow \mathcal{T}$ and a class of diagrams $\Delta \subseteq \operatorname{Diag}(\mathcal{T}, \Sigma)$. Suppose that the triple $(\mathcal{T}, \Sigma, \Delta)$ satisfies all the axioms of a triangulated category except possibly for the octahedral axiom. Then the following are equivalent:

(1) Base change. For any triangle $X \stackrel{\mu}{\rightarrow} Y \stackrel{\nu}{\rightarrow} Z \stackrel{\omega}{\rightarrow} \Sigma X \in \Delta$ and any morphism $\alpha$ : $Z^{\prime} \rightarrow Z$, there exists a commutative diagram

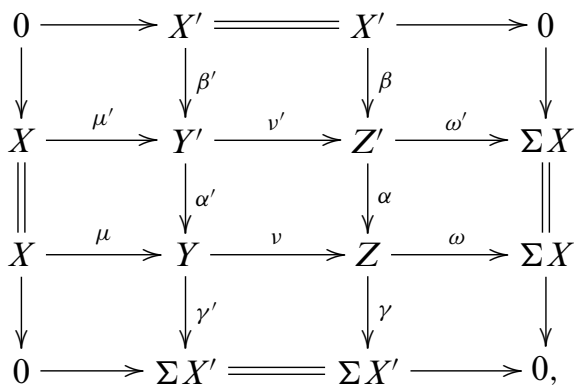

in which all horizontal and vertical diagrams are triangles in $\Delta$.

(2) Cobase change. For any triangle $X \stackrel{\mu}{\rightarrow} Y \stackrel{\nu}{\rightarrow} Z \stackrel{\omega}{\rightarrow} \Sigma X \in \Delta$ and any morphism $\beta$ : $X \rightarrow X^{\prime}$, there exists a commutative diagram

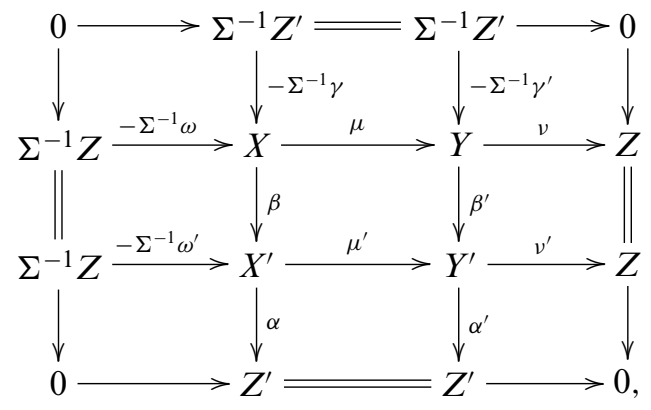

in which all horizontal and vertical diagrams are triangles in $\Delta$. 
(3) Octahedral axiom. For any two morphisms $\mu: X \rightarrow Y$ and $v: Y \rightarrow Z$, there exists a commutative diagram



in which all horizontal and the third vertical diagrams are triangles in $\Delta$.

Proper class of triangles. Let $\mathcal{T}=(\mathcal{T}, \Sigma, \Delta)$ be a triangulated category, where $\Sigma$ is the suspension functor and $\Delta$ is the triangulation.

A triangle $(T): X \stackrel{\mu}{\rightarrow} Y \stackrel{v}{\rightarrow} Z \stackrel{\omega}{\rightarrow} \Sigma X$ is called split if it is isomorphic to the triangle $X \stackrel{\left(\begin{array}{l}1 \\ 0\end{array}\right)}{\longrightarrow} X \oplus Z \stackrel{(0,1)}{\longrightarrow} Z \stackrel{0}{\longrightarrow} \Sigma X$. It is easy to see that $(T)$ is split if and only if $\mu$ is a section or $v$ is a retraction or $\omega=0$. The full subcategory of $\Delta$ comprising the split triangles will be denoted by $\Delta_{0}$. A class of triangles $\xi$ is closed under base change if for any triangle $X \stackrel{\mu}{\rightarrow} Y \stackrel{\nu}{\rightarrow} Z \stackrel{\omega}{\rightarrow} \Sigma X \in \xi$ and any morphism $\alpha: Z^{\prime} \rightarrow Z$ as in Proposition 2.1(1), the triangle $X \stackrel{\mu^{\prime}}{\rightarrow} Y^{\prime} \stackrel{v^{\prime}}{\rightarrow} Z^{\prime} \stackrel{\omega^{\prime}}{\rightarrow} \Sigma X$ is in $\xi$. Dually, a class of triangles $\xi$ is closed under cobase change if for any triangle $X \stackrel{\mu}{\rightarrow} Y \stackrel{\nu}{\rightarrow} Z \stackrel{\omega}{\rightarrow} \Sigma X \in \xi$ and any morphism $\beta: X \rightarrow X^{\prime}$ as in Proposition 2.1(2), the triangle $X^{\prime} \stackrel{\mu^{\prime}}{\rightarrow} Y^{\prime} \stackrel{\nu^{\prime}}{\rightarrow} Z \stackrel{\omega^{\prime}}{\rightarrow} \Sigma X^{\prime}$ is in $\xi$. A class of triangles $\xi$ is closed under suspensions if for any triangle $X \stackrel{\mu}{\rightarrow} Y \stackrel{\nu}{\rightarrow} Z \stackrel{\omega}{\rightarrow} \Sigma X \in \xi$ and any $i \in \mathbb{Z}$, the triangle

$$
\Sigma^{i} X \stackrel{(-1)^{i} \Sigma^{i} \mu}{\longrightarrow} \Sigma^{i} Y \stackrel{(-1)^{i} \Sigma^{i} v}{\longrightarrow} \Sigma^{i} Z \stackrel{(-1)^{i} \Sigma^{i} \omega}{\longrightarrow} \Sigma^{i+1} X
$$

is in $\xi$. A class of triangles $\xi$ is called saturated if in the situation of base change in Proposition 2.1, whenever the third vertical and the second horizontal triangles are in $\xi$, then the triangle $X \stackrel{\mu}{\rightarrow} Y \stackrel{\nu}{\rightarrow} Z \stackrel{\omega}{\rightarrow} \Sigma X$ is in $\xi$.

The following concept is inspired by the definition of an exact category [4].

A full subcategory $\xi \subseteq \operatorname{Diag}(\mathcal{T}, \Sigma)$ is called a proper class of triangles if the following conditions hold:

(i) $\xi$ is closed under isomorphisms, finite coproducts and $\Delta_{0} \subseteq \xi \subseteq \Delta$.

(ii) $\xi$ is closed under suspensions and is saturated.

(iii) $\xi$ is closed under base and co-base change.

For example, the class of split triangles $\Delta_{0}$ and the class of all triangles $\Delta$ in $\mathcal{T}$ are proper classes of triangles.

From now on, we fix a triangulated category $\mathcal{T}=(\mathcal{T}, \Sigma, \Delta)$ and a proper class of triangles $\xi$ in $\mathcal{T}$, where $\Sigma$ is the suspension functor and $\Delta$ is the triangulation.

Let $X \stackrel{\mu}{\rightarrow} Y \stackrel{\nu}{\rightarrow} Z \stackrel{\omega}{\rightarrow} \Sigma X$ be a triangle in $\xi$. The morphism $v: Y \rightarrow Z$ is called a $\xi$-proper epic and $\mu: X \rightarrow Y$ is called a $\xi$-proper monic; the morphism $\omega: Z \rightarrow \Sigma X$ is called a $\xi$-phantom map (see [3]). The class of $\xi$-phantom maps is denoted by $\operatorname{Ph}_{\xi}(\mathcal{T})$. In this case, $\mu$ is called the hokernel of $v$ and $v$ is called the hocokernel of $\mu$ (see [11]). 
PROPOSITION 2.2.

(1) If in the base change diagram in Proposition 2.1, the third horizontal triangle is in $\xi$, then the diagram $Y^{\prime} \stackrel{\left(\begin{array}{c}\alpha^{\prime} \\ -v^{\prime}\end{array}\right)}{\longrightarrow} Y \oplus Z^{\prime} \stackrel{(v, \alpha)}{\longrightarrow} Z \stackrel{\left(\Sigma \mu^{\prime}\right) \omega}{\longrightarrow} \Sigma Y^{\prime}$ is also in $\xi$.

(2) $\xi$ is saturated if and only if in the situation of cobase change in Proposition 2.1 the triangles $X^{\prime} \stackrel{\mu^{\prime}}{\rightarrow} Y^{\prime} \stackrel{\nu^{\prime}}{\rightarrow} Z \stackrel{\omega^{\prime}}{\rightarrow} \Sigma X^{\prime}$ and $X \stackrel{\beta}{\rightarrow} X^{\prime} \stackrel{\alpha}{\rightarrow} Z^{\prime} \stackrel{\gamma}{\rightarrow} \Sigma X$ are in $\xi$, then the triangle $X \stackrel{\mu}{\rightarrow} Y \stackrel{\nu}{\rightarrow} Z \stackrel{\omega}{\rightarrow} \Sigma X$ is also in $\xi$.

(3) If in the cobase change diagram in Proposition 2.1 the triangle $X \stackrel{\mu}{\rightarrow} Y \stackrel{\nu}{\rightarrow} Z \stackrel{\omega}{\rightarrow} \Sigma X$ is in $\xi$, then the diagram $X \stackrel{\left(\begin{array}{c}-\beta \\ \mu\end{array}\right)}{\longrightarrow} X^{\prime} \oplus Y \stackrel{\left(\mu^{\prime}, \beta^{\prime}\right)}{\longrightarrow} Y^{\prime} \stackrel{\omega v^{\prime}}{\longrightarrow} \Sigma X$ is also in $\xi$.

Proof.

(1) Consider the base change diagram in Proposition 2.1. Then the second horizontal triangle is in $\xi$ and [9, Axiom D'] implies that the diagram $Y^{\prime} \stackrel{\left({ }_{-v^{\prime}}\right)}{\longrightarrow} Y \oplus Z^{\prime} \stackrel{(v, \alpha)}{\longrightarrow}$ $Z \stackrel{\left(\Sigma \mu^{\prime}\right) \omega}{\longrightarrow} \Sigma Y^{\prime}$ is in $\Delta$. Also, we have the following commutative diagram:



in which all horizontal and vertical diagrams are in $\Delta$. Note that the second horizontal triangle is in $\Delta_{0}$, it follows from the saturation that the triangle $Y^{\prime} \stackrel{\left(\begin{array}{l}\alpha^{\prime} \\ -v^{\prime}\end{array}\right)}{\longrightarrow}$ $Y \oplus Z^{\prime} \stackrel{(\nu, \alpha)}{\longrightarrow} Z \stackrel{\left(\Sigma \mu^{\prime}\right) \omega}{\longrightarrow} \Sigma Y^{\prime}$ is in $\xi$.

(2) We only prove the 'only if' part, the proof of the 'if' part is similar.

By assumption and [9, Axiom D], we have the following commutative diagram:

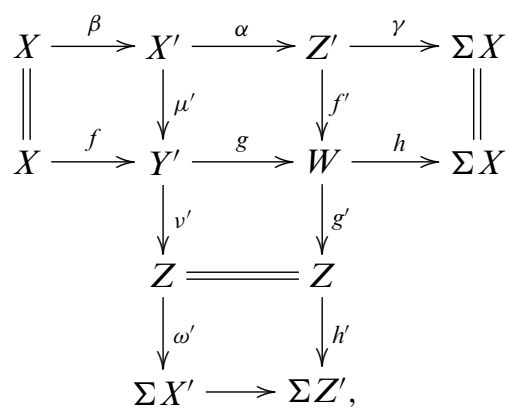

in which the first horizontal and the second vertical triangles are in $\xi$. So the third vertical triangle is in $\xi$ by cobase change. Now by analogy with the proof of (1), we have the triangle $X^{\prime} \stackrel{\left(\begin{array}{c}-\alpha \\ \mu^{\prime}\end{array}\right)}{\longrightarrow} Z^{\prime} \oplus Y^{\prime} \stackrel{\left(f^{\prime}, g^{\prime}\right)}{\longrightarrow} W \stackrel{\omega^{\prime} g^{\prime}}{\longrightarrow} \Sigma X^{\prime}$ in $\xi$. Consider the following commutative diagram: 


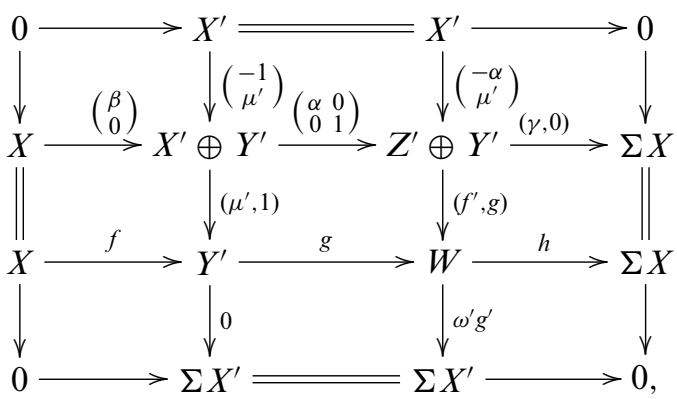

in which all horizontal and vertical diagrams are in $\Delta$. It follows from the saturation that the third horizontal triangle is in $\xi$. Again [9, Axiom D] yields a commutative diagram:

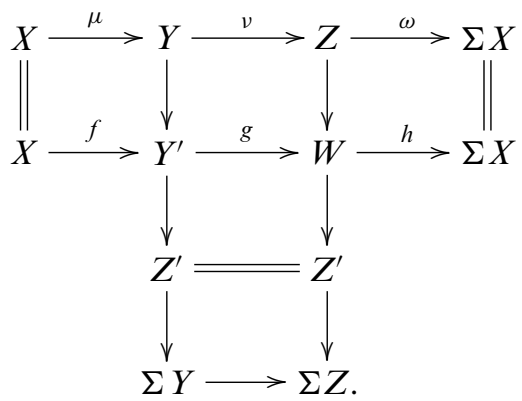

Thus, the base change implies that the triangle $X \stackrel{\mu}{\rightarrow} Y \stackrel{\nu}{\rightarrow} Z \stackrel{\omega}{\rightarrow} \Sigma X$ is in $\xi$.

(3) The proof is dual to that of (1).

Proposition 2.3. Given a commutative diagram:



in which all horizontal and vertical diagrams are in $\Delta$.

(1) If the third vertical triangle and the triangle $X \stackrel{\mu}{\rightarrow} Y \stackrel{v}{\rightarrow} Z \stackrel{\omega}{\rightarrow} \Sigma X$ are in $\xi$, then the triangle $X^{\prime} \stackrel{\mu^{\prime}}{\rightarrow} Y^{\prime} \stackrel{v^{\prime}}{\rightarrow} Z \stackrel{\omega^{\prime}}{\rightarrow} \Sigma X^{\prime}$ is also in $\xi$.

(2) If the second vertical triangle and the triangle $X^{\prime} \stackrel{\mu^{\prime}}{\rightarrow} Y^{\prime} \stackrel{v^{\prime}}{\rightarrow} Z \stackrel{\omega^{\prime}}{\rightarrow} \Sigma X^{\prime}$ are in $\xi$, then the third vertical triangle is also in $\xi$.

Proof. We will just prove (1), since (2) is dual. 
Note that the second vertical triangle is in $\xi$ by base change. Thus, Proposition 2.2(1) implies that the diagram $X^{\prime} \stackrel{\left(\begin{array}{c}\mu^{\prime} \\ -\beta^{\prime}\end{array}\right)}{\longrightarrow} Y^{\prime} \oplus X \stackrel{(\beta, \mu)}{\longrightarrow} Y \stackrel{\left(\Sigma \alpha^{\prime}\right) \gamma}{\longrightarrow} \Sigma X^{\prime}$ is also in $\xi$. But we also have the following commutative diagram:



in which all horizontal and vertical diagrams are in $\Delta$. By definition, the triangle $Y^{\prime} \oplus X \stackrel{\left(\begin{array}{ll}1 & 0 \\ 0 & \mu\end{array}\right)}{\longrightarrow} Y^{\prime} \oplus Y \stackrel{(0, v)}{\longrightarrow} Z \stackrel{\left(\begin{array}{c}0 \\ \omega\end{array}\right)}{\longrightarrow} \Sigma\left(Y^{\prime} \oplus X\right)$ is in $\xi$. Hence, Proposition 2.2(2) shows that the triangle $X^{\prime} \stackrel{-\mu^{\prime}}{\rightarrow} Y^{\prime} \stackrel{\nu^{\prime}}{\rightarrow} Z \stackrel{-\omega^{\prime}}{\rightarrow} \Sigma X^{\prime}$ is in $\xi$, and so $X^{\prime} \stackrel{\mu^{\prime}}{\rightarrow} Y^{\prime} \stackrel{\nu^{\prime}}{\rightarrow} Z \stackrel{\omega^{\prime}}{\rightarrow} \Sigma X^{\prime}$ is also in $\xi$.

Proposition 2.4. The class of $\xi$-proper monics is closed under compositions. Dually, the class of $\xi$-proper epics is closed under compositions.

Proof. This follows immediately from the saturation property by chasing the base and cobase change diagrams.

Proposition 2.5. Consider two morphisms, $\mu: X \rightarrow Y$ and $v: Y \rightarrow Z$.

(1) If $\nu \mu$ is a $\xi$-proper monic, then $\mu$ is a $\xi$-proper monic.

(2) If $\nu \mu$ is a $\xi$-proper epic, then $\nu$ is a $\xi$-proper epic.

Proof. This follows immediately from the base and cobase change diagrams.

Baer's Theory ([3]). Let $(T): X \stackrel{\mu}{\rightarrow} Y \stackrel{v}{\rightarrow} Z \stackrel{\omega}{\rightarrow} \Sigma X$ be a triangle in $\xi$. We call $\omega$ : $Z \rightarrow \Sigma X$ the characteristic class of $\Delta$, and usually we denote it by $\operatorname{ch}(T)=\omega$. Let $X, Z$ be two objects of $\mathcal{T}$, and consider the class $\xi^{*}(Z, X)$ of all triangles $X \stackrel{\mu}{\rightarrow} Y \stackrel{\nu}{\rightarrow} Z \stackrel{\omega}{\rightarrow}$ $\Sigma X$ in $\xi$. We define a relation in $\xi^{*}(Z, X)$ as follows: If $(T)_{i}: X \stackrel{\mu_{i}}{\rightarrow} Y_{i} \stackrel{\nu_{i}}{\rightarrow} Z \stackrel{\omega_{i}}{\rightarrow} \Sigma X$, $i=1,2$, are elements of $\xi^{*}(Z, X)$, then we define $(T)_{1} \sim(T)_{2}$ if there exists a morphism of triangles:



Obviously, $g$ is an isomorphism and $\sim$ is an equivalence relation on the class $\xi^{*}(Z, X)$. Using base and cobase change, it is easy to see that we can define (as in the case of the classical Baer's theory in an abelian category) a sum in the class 
$\xi(Z, X):=\xi^{*}(Z, X) / \sim$ in such a way that $\xi(Z, X)$ becomes an abelian group and $\xi(-,-): \mathcal{T}^{\text {op }} \times \mathcal{T} \rightarrow \mathcal{A}$ b becomes an additive bifunctor. Trivially, we have $c h$, which is an isomorphism of bifunctors: $\xi(-,-) \rightarrow \mathrm{Ph}_{\xi}(-, \Sigma-)$.

Definition 2.6. A pair of classes $(\mathcal{X}, \mathcal{Y})$ in a triangulated category $\mathcal{T}$ is a cotorsion pair with respect to $\xi$ if the following conditions hold:

(1) $\xi(X, Y)=0$ for all $X \in \mathcal{X}$ and $Y \in \mathcal{Y}$.

(2) If $\xi(X, Z)=0$ for all $X \in \mathcal{X}$, then $Z \in \mathcal{Y}$.

(3) If $\xi(Z, Y)=0$ for all $Y \in \mathcal{Y}$, then $Z \in \mathcal{X}$.

A cotorsion pair $(\mathcal{X}, \mathcal{Y})$ with respect to $\xi$ is said to have enough $\xi$-projectives if for any $T \in \mathcal{T}$ there is a triangle $Y \rightarrow X \rightarrow T \rightarrow \Sigma Y$ in $\xi$ where $X \in \mathcal{X}$ and $Y \in \mathcal{Y}$. We say it has enough $\xi$-injectives if for any $T \in \mathcal{T}$ there is a triangle $T \rightarrow Y \rightarrow X \rightarrow \Sigma T$ in $\xi$, where $X \in \mathcal{X}$ and $Y \in \mathcal{Y}$. If both of these hold, we say that $(\mathcal{X}, \mathcal{Y})$ is a complete cotorsion pair with respect to $\xi$.

3. Triangulated model structures with respect to $\xi$. First we define some terminology from the theory of model categories [7] and [8].

Note that if $\mathcal{T}$ is a triangulated category, then for any $T \in \mathcal{T}, 0 \rightarrow T$ is a $\xi$-proper monic and $T \rightarrow 0$ is a $\xi$-proper epic. Now suppose $\mathcal{T}$ has a model structure with respect to $\xi$ as defined in [7, Definition 1.1.3]. The three subcategories of $\mathcal{T}$ are called weak equivalences, cofibrations and fibrations respectively.

We say that $T$ is trivial if $0 \rightarrow T$ is a weak equivalence. By the 2-out-of-3 axiom and the fact that identity morphisms are always weak equivalences, this is equivalent to insisting that $T \rightarrow 0$ is a weak equivalence.

We say $T$ is cofibrant if $0 \rightarrow T$ is a cofibration.

We say $T$ is fibrant if $T \rightarrow 0$ is a fibration.

We say $T$ is trivially cofibrant if it is both trivial and cofibrant, i.e. $0 \rightarrow T$ is a trivial cofibration.

We say $T$ is trivially fibrant if it is both trivial and fibrant, i.e. $T \rightarrow 0$ is a trivial fibration.

We are now ready to define a triangulated model structure with respect to $\xi$ on $\mathcal{T}$.

DEFINITION 3.1. A triangulated model structure with respect to $\xi$ on $\mathcal{T}$ is a model structure in the sense of [7, Definition 1.1.3], in which each of the following holds.

(1) A morphism is a (trivial) cofibration if and only if it is a $\xi$-proper monic with a (trivially) cofibrant hocokernel.

(2) A morphism is a (trivial) fibration if and only if it is a $\xi$-proper epic with a (trivially) fibrant hokernel.

Let $\mathcal{T}$ be a triangulated category with a triangulated model structure with respect to $\xi$. Then by the 2-out-of-3 axiom, a morphism $g$ is a weak equivalence if and only if it has a factorization $g=p i$ with $i$ being a trivial cofibration and $p$ a trivial fibration.

DEFINITION 3.2. Given a triangulated category $\mathcal{T}$, by a thick subcategory of $\mathcal{T}$ we mean a class of objects $\mathcal{W}$ that is closed under direct summands such that if two out of three of the terms in a triangle of $\xi$ are in $\mathcal{W}$, so is the third.

The following theorem is contained in Theorem A, which shows that triangulated model structures compatible with $\xi$ gives us two complete cotorsion pairs with respect to $\xi$. 
THEOREM 3.3. Suppose $\mathcal{T}$ has a triangulated model structure with respect to $\xi$. Let $\mathcal{C}$ denote the full subcategory of cofibrant objects, $\mathcal{F}$ denote the full subcategory of fibrant objects, and $\mathcal{W}$ denote the full subcategory of trivial objects. Then,

(1) $\mathcal{W}$ is a thick subcategory of $\mathcal{T}$; and

(2) $(\mathcal{C}, \mathcal{F} \cap \mathcal{W})$ and $(\mathcal{C} \cap \mathcal{W}, \mathcal{F})$ are complete cotorsion pairs with respect to $\xi$.

Proof.

(1) It is clear that $\mathcal{W}$ is closed under retracts.

Given a triangle $X \stackrel{f}{\rightarrow} Y \stackrel{g}{\rightarrow} Z \stackrel{h}{\rightarrow} \Sigma X$ in $\xi$, we can write $g=p i$, where $p$ is a fibration and $i$ is a trivial cofibration. For $p$, there exists a triangle $X^{\prime} \stackrel{k}{\rightarrow} Y^{\prime} \stackrel{p}{\rightarrow}$ $Z \rightarrow \Sigma X^{\prime}$ in $\xi$ with $X^{\prime} \in \mathcal{F}$. Then [9, Axioms B' and E] yields the following commutative diagram:

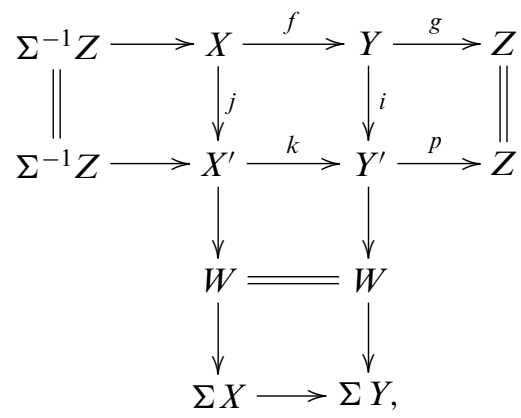

in which the third vertical triangle is in $\xi$. Note that Proposition 2.4 implies that $k j=$ if is a $\xi$-proper monic, it follows from Proposition 2.5 that $j$ is a $\xi$-proper monic. But $W \in \mathcal{C} \cap \mathcal{W}$, so $j$ is a trivial cofibration. Thus, if $X \in \mathcal{W}$ then $X^{\prime} \in \mathcal{W}$, and so $p$ is a trivial fibration and $g$ is a weak equivalence. This implies $Y \in \mathcal{W}$ if and only if $Z \in \mathcal{W}$. Similarly, if $Y, Z \in \mathcal{W}$, then $p$ is a trivial fibration. Thus, $X^{\prime} \in \mathcal{W}$, and hence $X \in \mathcal{W}$.

(2) We will prove that $(\mathcal{C}, \mathcal{F} \cap \mathcal{W})$ is a complete cotorsion pair with respect to $\xi$. The proof for $(\mathcal{C} \cap \mathcal{W}, \mathcal{F})$ is similar. We first show that $\xi(C, W)=0$ for all $C \in \mathcal{C}$ and $W \in \mathcal{F} \cap \mathcal{W}$. Indeed, an element of $\xi(C, W)$ is represented by a triangle $W \stackrel{i}{\rightarrow} X \stackrel{p}{\rightarrow} C \rightarrow \Sigma W$ in $\xi$. By assumption, $p$ is a trivial fibration. Since $C \in \mathcal{C}$, we can find a lift in the following commutative diagram:

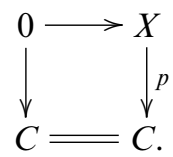

This lift defines the splitting of triangle, so $\xi(C, W) \cong \mathrm{Ph}_{\xi}(C, \Sigma W)=0$.

Now suppose $A$ is some object such that $\xi(A, W)=0$ for all $W \in \mathcal{F} \cap \mathcal{W}$. We must show that $A \in \mathcal{C}$. Since cofibrations in a triangulated model structure are the morphisms that have the left lifting property with respect to trivial fibrations, it suffices to show that, given a trivial fibration $p: X \rightarrow Y$ and a triangle $W \rightarrow X \stackrel{p}{\rightarrow}$ $Y \rightarrow \Sigma W$ in $\xi$ with $W \in \mathcal{F} \cap \mathcal{W}$, the $\operatorname{map} \mathcal{T}(A, X) \rightarrow \mathcal{T}(A, Y)$ is surjective. Note that $\mathcal{T}(A, X) \rightarrow \mathcal{T}(A, Y) \rightarrow \xi(A, W)$ is exact and $\xi(A, W)=0$, as desired.

Dually, suppose that $X$ is some object such that $\xi(C, X)=0$ for all $C \in \mathcal{C}$. We must show that $X \in \mathcal{F} \cap \mathcal{W}$. Since trivial fibrations in a triangulated model structure 
are the morphisms that have the right lifting property with respect to cofibrations, it suffices to show that, given a cofibration $i: A \rightarrow B$ and a triangle $A \stackrel{i}{\rightarrow} B \rightarrow$ $C \rightarrow \Sigma A$ in $\xi$ with $C \in \mathcal{C}$, the map $\mathcal{T}(B, X) \rightarrow \mathcal{T}(A, X)$ is surjective. Note that $\mathcal{T}(B, X) \rightarrow \mathcal{T}(A, X) \rightarrow \xi(C, X)$ is exact and $\xi(C, X)=0$, as claimed.

Finally, suppose $T$ is an object of $\mathcal{T}$. Then, by factoring the morphism $0 \rightarrow T$ into a cofibration followed by a trivial fibration, we find a trivial fibration $C \stackrel{p}{\rightarrow} T$, where $C \in \mathcal{C}$. This gives us a triangle $W \rightarrow C \rightarrow T \rightarrow \Sigma W$ in $\xi$ with $W \in \mathcal{F} \cap \mathcal{W}$. Thus, the cotorsion pair $(\mathcal{C}, \mathcal{F} \cap \mathcal{W})$ has enough $\xi$-projectives. Similarly, by factoring the morphism $T \rightarrow 0$ into a cofibration followed by a trivial fibration, we get a cofibration $T \stackrel{i}{\rightarrow} W^{\prime}$, where $W^{\prime} \in \mathcal{F} \cap \mathcal{W}$. This gives us a triangle $T \rightarrow W^{\prime} \rightarrow$ $C^{\prime} \rightarrow \Sigma T$ in $\xi$ with $C^{\prime} \in \mathcal{C}$. Hence, the cotorsion pair $(\mathcal{C}, \mathcal{F} \cap \mathcal{W})$ has enough $\xi$-injectives. This completes the proof.

4. Construction of triangulated model structures. Given classes $\mathcal{C}, \mathcal{F}$ and $\mathcal{W}$ such that $\mathcal{W}$ is a thick subcategory of $\mathcal{T}$ and $(\mathcal{C}, \mathcal{F} \cap \mathcal{W})$ and $(\mathcal{C} \cap \mathcal{W}, \mathcal{F})$ are complete cotorsion pairs with respect to $\xi$. The aim of this section is to give a method of constructing triangulated model structures with respect to $\xi$. First we define

cofibration $=\mathrm{a} \xi$-proper monic with hocokernel in $\mathcal{C}$,

trivial cofibration $=$ a $\xi$-proper monic with hocokernel in $\mathcal{C} \cap \mathcal{W}$,

fibration $=$ a $\xi$-proper epic with hokernel in $\mathcal{F}$,

trivial fibration $=\mathrm{a} \xi$-proper epic with hokernel in $\mathcal{F} \cap \mathcal{W}$,

weak equivalence $=$ a composition of a trivial cofibration followed by a trivial fibration.

Since $(\mathcal{C}, \mathcal{F} \cap \mathcal{W})$ is a cotorsion pair with respect to $\xi, 0$ must be in $\mathcal{C} \cap \mathcal{F} \cap \mathcal{W}$. Hence, the identity morphism, or any isomorphism, is both trivial fibration and trivial cofibration. Thus, every trivial cofibration or trivial fibration is a weak equivalence. In what follows, we check that these structures satisfy the lifting, factorization, retract and 2-out-of-3 axioms for a triangulated model structure with respect to $\xi$.

LEMMA 4.1. Cofibrations (resp. trivial cofibrations) have the left lifting property with respect to trivial fibrations (resp. fibrations).

Proof. Suppose we have the following commutative square:

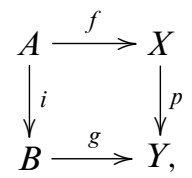

where $i: A \rightarrow B$ is a cofibration and $p: X \rightarrow Y$ is a trivial fibration. Then there exist triangles $A \stackrel{i}{\rightarrow} B \stackrel{j}{\rightarrow} C \rightarrow \Sigma A \in \xi$ with $C \in \mathcal{C}$ and $W \rightarrow X \stackrel{p}{\rightarrow} Y \rightarrow \Sigma W \in \xi$ with $W \in \mathcal{F} \cap \mathcal{W}$. This diagram induces a commutative diagram with exact rows and columns: 


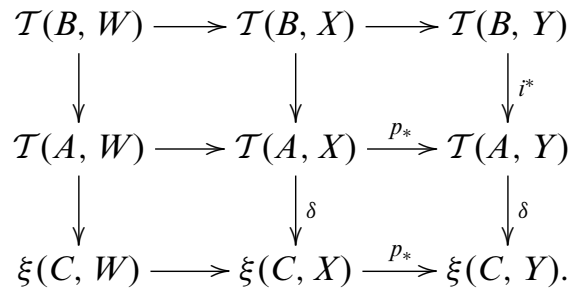

The morphism $f$ is an element of $\mathcal{T}(A, X)$ such that $p_{*} f=i^{*} g$. Thus, $p_{*} \delta f=\delta p_{*} f=0$. But $(\mathcal{C}, \mathcal{F} \cap \mathcal{W})$ is a cotorsion pair, so $\xi(C, W)=0$, and hence $\delta f=0$. It follows that there exists a morphism $h^{\prime}: B \rightarrow X$ such that $h^{\prime} i=f$. On the other hand, since $i^{*}\left(p h^{\prime}-\right.$ $g)=\left(p h^{\prime}-g\right) i=0$, there is a morphism $\alpha: C \rightarrow Y$ such that $\alpha j=p h^{\prime}-g$. The image of $\alpha$ in $\mathrm{Ph}_{\xi}(C, \Sigma W) \cong \xi(C, W)=0$ is obviously 0 , so there exists a morphism $\beta: C \rightarrow$ $X$ such that $p \beta=\alpha$. Set $h=h^{\prime}-\beta j$. Then $h i=f$ and $p h=g$, as desired.

LEMMA 4.2. Cofibrations, trivial cofibrations and fibrations, trivial fibrations are all subcategories of $\mathcal{T}$.

Proof. We just check that cofibrations are subcategories; the other cases are proved in a similar fashion.

Assume $j i$ is defined with $i$ and $j$ cofibrations. Then $j i$ is a $\xi$-proper monic by Proposition 2.4. Thus, [9, AxiomD] yields the following commutative diagram:



in which the first horizontal and the second vertical triangles are in $\xi$ with $C, C^{\prime \prime} \in$ $\mathcal{C}$. But $q l=p$ is a $\xi$-proper epic, and Proposition 2.5 implies that the third vertical triangle is in $\xi$. Note that the second horizontal triangle is in $\xi$ and $C^{\prime} \in \mathcal{C}$, so $j i$ is a cofibration.

LEMMA 4.3. The classes of cofibrations, trivial cofibrations and fibrations, trivial fibrations are all closed under retracts.

Proof. We just check that the class of cofibrations is closed under retracts; the other cases are proved in a similar fashion. Let

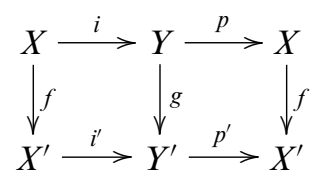

be a commutative diagram, where $f$ is a retract of $g$. 
Assume that $g$ is a cofibration. Then we have the following commutative diagram:

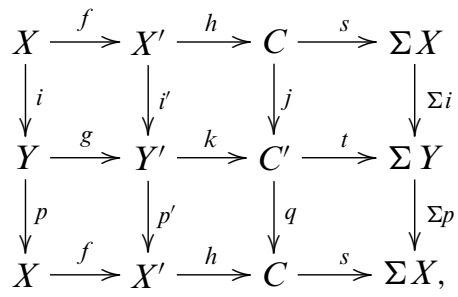

in which all the horizontal diagrams are in $\Delta$ and the second horizontal triangle is in $\xi$ with $C^{\prime} \in \mathcal{C}$. Note that Proposition 2.4 implies that $i^{\prime} f=g i$ is a $\xi$-proper monic, it follows from Proposition 2.5 that $f$ is a $\xi$-proper monic and the first horizontal triangle is in $\xi$. On the other hand, $q j h=q k i^{\prime}=h p^{\prime} i^{\prime}=h$ and $s q j=(\Sigma p) t j=\Sigma(p i) s=s$. We have the following morphism of triangles:

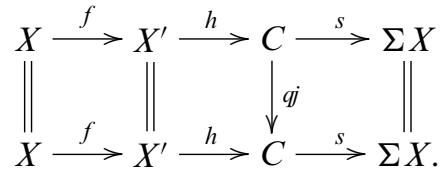

Hence, $q j$ is an isomorphism and $C$ is isomorphic to a summand of $C^{\prime}$, and so $C \in \mathcal{C}$. This means that $f$ is a cofibration.

We now investigate the factorization axiom.

LEMMA 4.4. Every morphism $f$ in $\mathcal{T}$ can be factored as $f=p i=q j$, where $p$ is a fibration, $i$ is a trivial cofibration and $q$ is a trivial fibration, $j$ is a cofibration.

Proof. We first show that any $\xi$-proper monic $f: X \rightarrow Y$ can be factored as $f=q j$, where $q$ is a trivial fibration and $j$ is a cofibration. Indeed, there is a triangle $X \stackrel{f}{\rightarrow} Y \rightarrow Z \rightarrow \Sigma X$ in $\xi$. Since $(\mathcal{C}, \mathcal{F} \cap \mathcal{W})$ is a complete cotorsion pair with respect to $\xi$, there exists a triangle $W \rightarrow C \rightarrow Z \rightarrow \Sigma W$ in $\xi$ with $C \in \mathcal{C}$ and $W \in \mathcal{F} \cap \mathcal{W}$. Applying base change for the triangle $X \stackrel{f}{\rightarrow} Y \rightarrow Z \rightarrow \Sigma X$ along $C \rightarrow Z$, we get the following commutative diagram:



in which the second horizontal and the second vertical triangles are in $\xi$. Thus, $f=q j$, where $q$ is a trivial fibration and $j$ is a cofibration.

We now show that any $\xi$-proper epic $f: Y \rightarrow Z$ can be factored as $f=q$, where $q$ is a trivial fibration and $j$ is a cofibration. Indeed, there exists a triangle $X \rightarrow Y \stackrel{f}{\rightarrow}$ $Z \rightarrow \Sigma X$ in $\xi$. Since $(\mathcal{C}, \mathcal{F} \cap \mathcal{W})$ is a complete cotorsion pair with respect to $\xi$, there 
is a triangle $X \rightarrow W \rightarrow C \rightarrow \Sigma X$ in $\xi$ with $C \in \mathcal{C}$ and $W \in \mathcal{F} \cap \mathcal{W}$. Applying cobase change for the triangle $\Sigma^{-1} Z \rightarrow X \rightarrow Y \stackrel{f}{\rightarrow} Z$ along $X \rightarrow W$, we get the following commutative diagram:

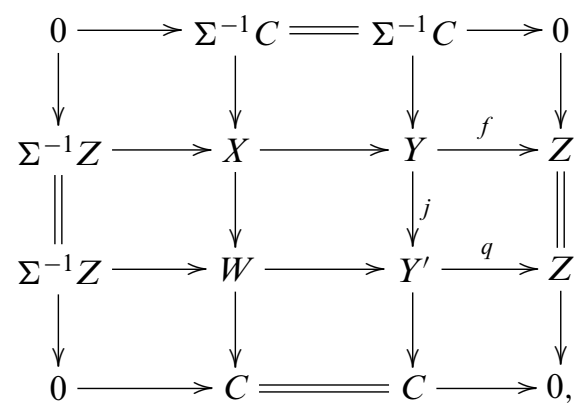

in which the triangles $W \rightarrow Y^{\prime} \stackrel{q}{\rightarrow} Z \rightarrow \Sigma W$ and $Y \stackrel{j}{\rightarrow} Y^{\prime} \rightarrow C \rightarrow \Sigma Y$ are in $\xi$. Hence, $f=q j$ with $q$ is a trivial fibration and $j$ is a cofibration.

Now suppose $f: A \rightarrow B$ is an arbitrary morphism in $\mathcal{T}$. Then we can factor $f$ as $A \stackrel{\left(\begin{array}{l}1 \\ 0\end{array}\right)}{\rightarrow} A \oplus B \stackrel{(f, 1)}{\rightarrow} B$. Since $\left(\begin{array}{l}1 \\ 0\end{array}\right)$ is a split monomorphism, it is a $\xi$-proper monic. Similarly, since $(f, 1)$ is a split epimorphism, it is a $\xi$-proper epic. Hence, we can write $(f, 1)=q^{\prime} j^{\prime}$, where $q^{\prime}$ is a trivial fibration and $j^{\prime}$ is a cofibration. But the composite $j^{\prime}\left(\begin{array}{l}1 \\ 0\end{array}\right)$ is a $\xi$-proper monic, so we can write $j^{\prime}\left(\begin{array}{l}1 \\ 0\end{array}\right)=q^{\prime \prime} j$, where $q^{\prime \prime}$ is a trivial fibration and $j$ is a cofibration. Let $q=q^{\prime} q^{\prime \prime}$. Then $f=q j$, where $q$ is a trivial fibration by Lemma 4.2, $j$ is a cofibration.

The proof of the factorization $f=p i$, where $p$ is a fibration and $i$ is a trivial cofibration, is similar.

The following lemma gives a better understanding of weak equivalences in $\mathcal{T}$.

Lemma 4.5. Suppose $i: A \rightarrow B$ is a $\xi$-proper monic. Then $i$ is a weak equivalence if and only if there exists a triangle $A \stackrel{i}{\rightarrow} B \rightarrow W \rightarrow \Sigma A$ in $\xi$ with $W \in \mathcal{W}$. In particular, a morphism that is both a cofibration and a weak equivalence is a trivial cofibration. Dually, a $\xi$-proper epic $p: X \rightarrow Y$ is a weak equivalence if and only if there is a triangle $W \rightarrow X \stackrel{p}{\rightarrow} Y \rightarrow \Sigma W$ in $\xi$ with $W \in \mathcal{W}$, so a morphism that is both a fibration and a weak equivalence is a trivial fibration.

Proof. We just prove one of the statements, since the other is dual.

Factor $i=p j$, where $p$ is a trivial fibration and $j$ is a cofibration. Then [9, Axiom D] yields the following commutative diagram:




in which both the first and the second horizontal triangles and the triangle $W^{\prime \prime} \rightarrow$ $B^{\prime} \stackrel{p}{\rightarrow} B \rightarrow \Sigma W^{\prime \prime}$ are in $\xi$ with $W^{\prime} \in \mathcal{C}$ and $W^{\prime \prime} \in \mathcal{F} \cap \mathcal{W}$. But Proposition 2.4 implies that $q k=l p$ is a $\xi$-proper epic, it follows from Proposition 2.5 that $q$ is a $\xi$-proper epic and the triangle $W^{\prime \prime} \rightarrow W^{\prime} \stackrel{q}{\rightarrow} W \rightarrow \Sigma W^{\prime \prime}$ is in $\xi$. Since $\mathcal{W}$ is a thick subcategory and $W^{\prime \prime} \in \mathcal{W}$, we get $W \in \mathcal{W}$ if and only if $W^{\prime} \in \mathcal{W}$. In particular, if $W \in \mathcal{W}$ then $W^{\prime} \in \mathcal{W}$, and so $j$ is a trivial cofibration. This forces $i$ to be a weak equivalence. Conversely, if $i$ is a weak equivalence then we can take $j$ to be a trivial cofibration. This means $W^{\prime} \in \mathcal{W}$, and hence $W \in \mathcal{W}$.

LEMMA 4.6. Weak equivalences are closed under retracts.

Proof. Suppose $f$ is a retract of $g$ and we have a commutative diagram:

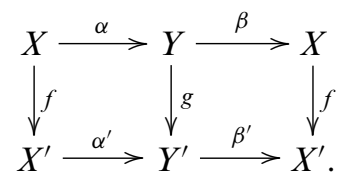

Since we have factorizations, we can write $f=p i$ and $g=q j$, where $q, p$ are trivial fibrations and $i, j$ are cofibrations. Then we have two commutative diagrams:



It follows from Lemma 4.1 that there are $\alpha^{\prime \prime}: X^{\prime \prime} \rightarrow Y^{\prime \prime}$ and $\beta^{\prime \prime}: Y^{\prime \prime} \rightarrow X^{\prime \prime}$ such that $\alpha^{\prime \prime} i=j \alpha, \alpha^{\prime} p=q \alpha^{\prime \prime}$ and $\beta^{\prime \prime} j=i \beta, p \beta^{\prime \prime}=\beta^{\prime} q$. Since $i$ is a cofibration, there exists a triangle $X \stackrel{i}{\rightarrow} X^{\prime \prime} \stackrel{\tau}{\rightarrow} C \rightarrow \Sigma X$ in $\xi$ with $C \in \mathcal{C}$. Note that $\beta^{\prime \prime} \alpha^{\prime \prime} i=i$, it follows from [9, Axiom B] that there is $h: C \rightarrow C$ such that $X^{\prime \prime} \stackrel{\left(\beta^{\prime \prime} \alpha^{\prime \prime}\right)}{\rightarrow} X^{\prime \prime} \oplus C \stackrel{(\tau, h)}{\rightarrow} C \stackrel{0}{\rightarrow} \Sigma X^{\prime \prime}$ is a triangle. But this triangle is split, so it is isomorphic to the triangle $X^{\prime \prime} \stackrel{(1)}{\rightarrow} X^{\prime \prime} \oplus C \stackrel{(0,1)}{\rightarrow}$ $C \stackrel{0}{\rightarrow} \Sigma X^{\prime \prime}$. This implies that $\beta^{\prime \prime} \alpha^{\prime \prime} \cong 1_{X^{\prime \prime}}$, and so $p$ is a retract of $q$, and $i$ is a retract of $j$. If $g$ is a weak equivalence, then $j$ is a trivial cofibration by Lemma 4.5, and so $i$ is also a trivial cofibration by Lemma 4.3. Therefore, $f$ is a weak equivalence, as desired.

We now begin the investigation of weak equivalences.

LEMMA 4.7. Weak equivalences are closed under compositions.

Proof. By Lemma 4.2, it suffices to show that if $p: X \rightarrow Y$ is a trivial fibration and $i: Y \rightarrow Z$ is a trivial cofibration, then $i p$ is a weak equivalence. Let us factor $i p=q j$, where $j$ is a cofibration and $q$ is a trivial fibration. Consider the triangles $X \stackrel{j}{\rightarrow}$ $X^{\prime} \stackrel{j^{\prime}}{\rightarrow} C \stackrel{j^{\prime \prime}}{\rightarrow} \Sigma X, \quad Y \stackrel{i}{\rightarrow} Z \stackrel{i^{\prime}}{\rightarrow} C^{\prime} \stackrel{i^{\prime \prime}}{\rightarrow} \Sigma Y$ with $C \in \mathcal{C}, C^{\prime} \in \mathcal{C} \cap \mathcal{W}$ and the triangles $F \stackrel{p^{\prime \prime}}{\rightarrow} X \stackrel{p}{\rightarrow} Y \stackrel{p^{\prime}}{\rightarrow} \Sigma F, F^{\prime} \stackrel{q^{\prime \prime}}{\rightarrow} X^{\prime} \stackrel{q}{\rightarrow} Z \stackrel{q^{\prime}}{\rightarrow} \Sigma F^{\prime}$ with $F, F^{\prime} \in \mathcal{F} \cap \mathcal{W}$. Using that $\Sigma$ is an automorphism and the $3 \times 3$ Lemma, the commutative square on the top left corner below is embedded in a diagram: 


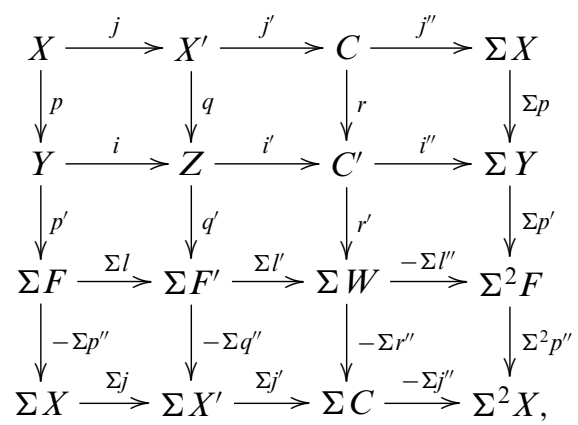

which is commutative except the lower right square, which anti-commutes and where all the rows and columns are in $\Delta$. Then we have the following diagram:



which is commutative except the lower right square, which anti-commutes. We now show that the first horizontal and the third vertical triangles in the above diagram are in $\xi$. First, Proposition 2.4 implies that $q^{\prime \prime} l=j p^{\prime \prime}$ is a $\xi$-proper monic, it follows from Proposition 2.5 that $l$ is a $\xi$-proper monic and the triangle $F \stackrel{l}{\rightarrow} F^{\prime} \stackrel{l^{\prime}}{\rightarrow} W \stackrel{l^{\prime \prime}}{\rightarrow} \Sigma F$ is in $\xi$. But $F, F^{\prime} \in \mathcal{W}$ and $\mathcal{W}$ is a thick subcategory, so $W \in \mathcal{W}$. Next, Proposition 2.4 implies that $r j^{\prime}=i^{\prime} q$ is a $\xi$-proper epic, it follows from Proposition 2.5 that $r$ is a $\xi$-proper epic and the triangle $W \stackrel{r^{\prime \prime}}{\rightarrow} C \stackrel{r}{\rightarrow} C^{\prime} \stackrel{r^{\prime}}{\rightarrow} \Sigma W$ is in $\xi$. But $C^{\prime}, W \in \mathcal{W}$ and $\mathcal{W}$ is a thick subcategory, so $C \in \mathcal{W}$, it follows that $C \in \mathcal{C} \cap \mathcal{W}$. Consequently, $i p=q j$ is a weak equivalence.

We now begin the investigation of 2-out-of-3 axiom.

LemMa 4.8. Suppose that $f i=j$, where $i$ and $j$ are trivial cofibrations. Then $f$ is a weak equivalence. Dually, if of $=p$, where $p$ and $q$ are trivial fibrations, then $f$ is a weak equivalence.

Proof. We just prove one of the statements, since the other is dual.

Suppose $f i=j$. By factoring $f=p k$, where $k$ is a trivial cofibration and $p$ is a fibration, we can reduce the case that $f$ is a fibration, and, in particular, a $\xi$-proper epic. Then [9, Axiom D] yields the following commutative diagram: 


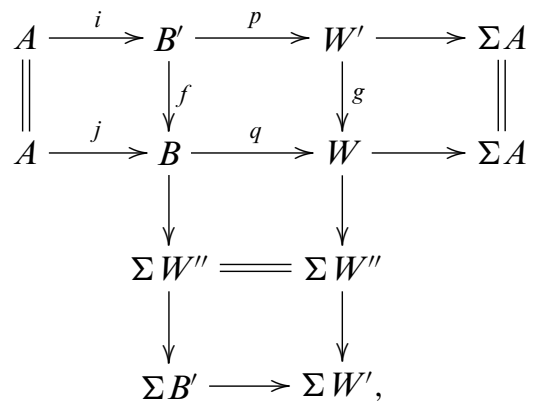

in which both the first and the second horizontal triangles and the triangle $W^{\prime \prime} \rightarrow$ $B^{\prime} \stackrel{f}{\rightarrow} B \rightarrow \Sigma W^{\prime \prime}$ are in $\xi$ with $W, W^{\prime} \in \mathcal{C} \cap \mathcal{W}$. Note that Proposition 2.4 implies that $g p=q f$ is a $\xi$-proper epic, it follows from Proposition 2.5 that $g$ is a $\xi$-proper epic and the triangle $W^{\prime \prime} \rightarrow W^{\prime} \stackrel{g}{\rightarrow} W \rightarrow \Sigma W^{\prime \prime}$ is in $\xi$. Since $W, W^{\prime} \in \mathcal{W}$ and $\mathcal{W}$ is a thick subcategory, $W^{\prime \prime} \in \mathcal{W}$. Hence, Lemma 4.5 implies that $f$ is a weak equivalence.

Lemma 4.9. Suppose that $p i=j$, where $p$ is a trivial fibration and $j$ is a trivial cofibration. Then $i$ is a weak equivalence. Dually, if $q j=p$, where $p$ is a trivial fibration and $j$ is a trivial cofibration, then $q$ is a weak equivalence.

Proof. Again, we will just prove one of the statements, since the other is dual.

By assumption and [9, Axiom D], we have the following commutative diagram:

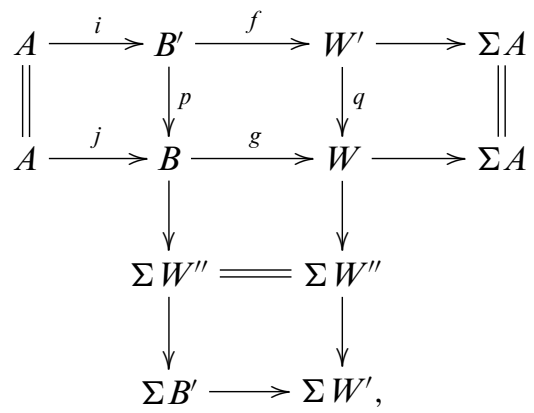

in which the second horizontal triangle and the triangle $W^{\prime \prime} \rightarrow B^{\prime} \stackrel{p}{\rightarrow} B \rightarrow \Sigma W^{\prime \prime}$ are in $\xi$ with $W \in \mathcal{C} \cap \mathcal{W}$ and $W^{\prime \prime} \in \mathcal{F} \cap \mathcal{W}$. But Proposition 2.4 implies that $q f=g p$ is a $\xi$-proper epic, it follows from Proposition 2.5 that $q$ is a $\xi$-proper epic and the triangle $W^{\prime \prime} \rightarrow W^{\prime} \stackrel{q}{\rightarrow} W \rightarrow \Sigma W^{\prime \prime}$ is in $\xi$. Since $W, W^{\prime \prime} \in \mathcal{W}$ and $\mathcal{W}$ is a thick subcategory, $W^{\prime} \in \mathcal{W}$. Note that $i$ is a $\xi$-proper monic by Proposition 2.5, it follows from Lemma 4.5 that $i$ is a weak equivalence.

Lemma 4.10. Suppose that if $=i$, where $i$ and $j$ are trivial cofibrations. Then $f$ is a weak equivalence. Dually, if $f q=p$, where $p$ and $q$ are trivial fibrations, then $f$ is a weak equivalence.

Proof. We just prove one of the statements, since the other is dual.

By assumption and [9, Axioms B' and E], we have the following commutative diagram: 


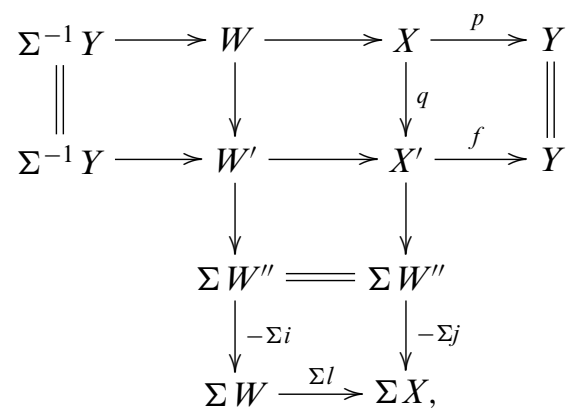

in which the triangles $W \rightarrow X \stackrel{p}{\rightarrow} Y \rightarrow \Sigma W$ and $W^{\prime \prime} \rightarrow X \stackrel{q}{\rightarrow} X^{\prime} \rightarrow \Sigma W^{\prime \prime}$ are in $\xi$ with $W, W^{\prime \prime} \in \mathcal{F} \cap \mathcal{W}$. But $l i=j$ is a $\xi$-proper monic and $f q=p$ is a $\xi$-proper epic, it follows from Proposition 2.5 that the triangles $W^{\prime} \rightarrow X^{\prime} \stackrel{f}{\rightarrow} Y \rightarrow \Sigma W^{\prime}$ and $W^{\prime \prime} \stackrel{i}{\rightarrow}$ $W \rightarrow W^{\prime} \rightarrow \Sigma W^{\prime \prime}$ are in $\xi$. Since $W, W^{\prime \prime} \in \mathcal{W}$ and $\mathcal{W}$ is a thick subcategory, $W^{\prime} \in \mathcal{W}$. Thus, Lemma 4.5 implies that $f$ is a weak equivalence.

Lemma 4.11. Suppose $f$ and $g$ are composable maps. Then if two of $f, g$ and $g f$ are weak equivalences, so is the third.

Proof. It follows from Lemma 4.2 that weak equivalences form a subcategory.

First we show that if $g f: X \rightarrow Z$ and $g: Y \rightarrow Z$ are weak equivalences, so is $f$. By factoring $f$ into a cofibration followed by a trivial fibration, we see that we may as well assume $f: X \rightarrow Y$ is a cofibration. Since $g$ is a weak equivalence, we can factor $g=p i$, where $i$ is a trivial cofibration and $p$ is a trivial fibration. We can then factor if $=q j$, where $j$ is a cofibration and $q$ is a trivial fibration. This gives us a commutative diagram:

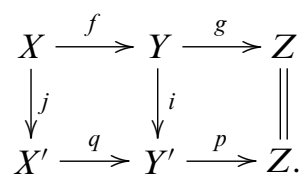

On the other hand, $g f$ is also a weak equivalence, so we can factor $g f=r k$, where $k$ is a trivial cofibration and $r$ is a trivial fibration. This yields a commutative diagram:

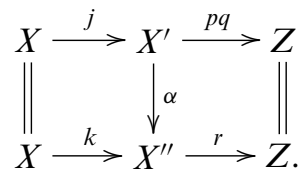

Here the morphism $\alpha$ exists because we can lift in the following commutative square:

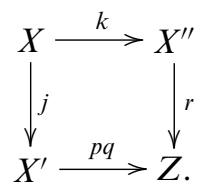

Note that we have $p q=r \alpha$, where $p q$ and $r$ are trivial fibrations. Hence, Lemma 4.8 shows that $\alpha$ is a weak equivalence. We can therefore factor $\alpha=s l$, where $s$ is a trivial fibration and $l$ is a trivial cofibration. We then have $s(l j)=k$, where $s$ is a trivial 
fibration and $k$ is a trivial cofibration. So Lemmas 4.9 and 4.5 imply that $l j$ is a trivial cofibration. Since $l$ is a trivial cofibration, Lemmas 4.10 and 4.5 imply that $j$ is a trivial cofibration. Therefore, $q j=$ if is a weak equivalence. This means that both if and $i$ are trivial cofibrations. Hence, Lemma 4.10 implies that $f$ is a weak equivalence.

Similarly, we can show that if $g f: X \rightarrow Z$ and $f: X \rightarrow Y$ are weak equivalences, then $g$ is also a weak equivalence.

We can now obtain a triangulated model structure with respect to $\xi$. The precise statement of our result follows, and is contained in Theorem A.

THEOREM 4.12. Given classes $\mathcal{W}, \mathcal{C}$ and $\mathcal{F}$ such that $\mathcal{W}$ is a thick subcategory of $\mathcal{T}$ and $(\mathcal{C}, \mathcal{F} \cap \mathcal{W})$ and $(\mathcal{C} \cap \mathcal{W}, \mathcal{F})$ are complete cotorsion pairs with respect to $\xi$, there is a unique triangulated model structure with respect to $\xi$ on $\mathcal{T}$ such that $\mathcal{W}$ is the class of trivial objects, $\mathcal{C}$ is the class of cofibrant objects and $\mathcal{F}$ is the class of fibrant objects.

Proof. By Lemma 4.11, weak equivalences satisfy 2-out-of-3 axiom. By Lemmas 4.3 and 4.6, the classes of weak equivalences, cofibrations and fibrations are all closed under retracts. By Lemma 4.1, cofibrations (resp. trivial cofibrations) have the left lifting property with respect to trivial fibrations (resp. fibrations). By Lemma 4.4, any morphism in $\mathcal{T}$ satisfies factorization axiom. Thus, we obtain a triangulated model structure with respect to $\xi$.

5. Applications. It is well known that if $R$ is a Gorenstein ring, then the class $\mathcal{W}$ of modules of finite projective dimension coincides with the class of modules of finite injective dimension. Further, the class of Gorenstein projective $R$-modules and $\mathcal{W}, \mathcal{W}$ and the class of Gorenstein injective $R$-modules form complete cotorsion pairs in the category of $R$-modules. Thus, Hovey [7, Section 8] constructed two model structures on the category of complexes of $R$-modules by applying [7, Theorem 2.2] to Gorenstein rings. As an application of the results in the above sections, we construct two triangulated model structures over a Gorenstein triangulated category. This result depends on Asadollahi and Salarian's work [1].

An object $P \in \mathcal{T}$ (resp. $I \in \mathcal{T}$ ) is called $\xi$-projective (resp. $\xi$-injective) if for any triangle $X \rightarrow Y \rightarrow Z \rightarrow \Sigma X$ in $\xi$, the induced sequence $0 \rightarrow \mathcal{T}(P, X) \rightarrow \mathcal{T}(P, Y) \rightarrow$ $\mathcal{T}(P, Z) \rightarrow 0$ (resp. $0 \rightarrow \mathcal{T}(Z, I) \rightarrow \mathcal{T}(Y, I) \rightarrow \mathcal{T}(X, I) \rightarrow 0)$ is exact in the category $\mathcal{A}$ b. The symbol $\mathcal{P}(\xi)$ (resp. $\mathcal{I}(\xi))$ will denote the full subcategory of $\xi$-projective (resp. $\xi$-injective) objects of $\mathcal{T}$. $\mathcal{T}$ is said to have enough $\xi$-projectives if for any object $X \in \mathcal{T}$ there exists a triangle $K \rightarrow P \rightarrow X \rightarrow \Sigma K$ in $\xi$ with $P \in \mathcal{P}(\xi)$. Dually, one defines when $\mathcal{T}$ has enough $\xi$-injectives.

In what follows, $\mathcal{T}$ will always be a triangulated category with enough $\xi$-projectives and $\xi$-injectives.

We define inductively the $\xi$-projective dimension $\xi$-pd $T$ of an object $T \in \mathcal{T}$ as follows. If $T \in \mathcal{P}(\xi)$, then $\xi$-pd $T=0$. Next, if $\xi$-pd $T>0$ define $\xi$-pd $T \leqslant n$ if there exists a triangle $K \rightarrow P \rightarrow T \rightarrow \Sigma K$ in $\xi$ with $P \in \mathcal{P}(\xi)$ and $\xi-p d K \leqslant n-1$. Finally, define $\xi-\operatorname{pd} T=n$ if $\xi-\operatorname{pd} T \leqslant n$ and $\xi-\operatorname{pd} T \not \leq n-1$. Of course, we set $\xi-\operatorname{pd} T=\infty$ if $\xi-\operatorname{pd} T \neq n$ for any $n \geqslant 0$. Dually, one defines $\xi$-id $T$ of an object $T \in \mathcal{T}$.

LeMma 5.1. $(\mathcal{P}(\xi), \mathcal{T})$ and $(\mathcal{T}, \mathcal{I}(\xi))$ are complete cotorsion pairs with respect to $\xi$.

Proof. This follows directly from assumptions. 
Following [1], a $\xi$-exact complex $X$ is a diagram $\cdots \rightarrow X_{n+1} \stackrel{d_{n+1}}{\rightarrow} X_{n} \stackrel{d_{n}}{\rightarrow} X_{n-1} \rightarrow \cdots$ in $\mathcal{T}$ such that for all integers $n$, there exist triangles $K_{n+1} \stackrel{g_{n}}{\rightarrow} X_{n} \stackrel{f_{n}}{\rightarrow} K_{n} \stackrel{h_{n}}{\rightarrow} \Sigma K_{n+1}$ in $\xi$, and the differential $d_{n}$ is defined as $d_{n}=g_{n-1} f_{n}$ for any integer $n$.

A triangle $X \rightarrow Y \rightarrow Z \rightarrow \Sigma X$ in $\xi$ is called $\mathcal{T}(-, \mathcal{P}(\xi)$ )-exact if for any $Q \in \mathcal{P}(\xi)$, the induced complex $0 \rightarrow \mathcal{T}(Z, Q) \rightarrow \mathcal{T}(Y, Q) \rightarrow \mathcal{T}(X, Q) \rightarrow 0$ is exact in $\mathcal{A}$ b.

A complete $\xi$-exact complex $X$ is a diagram $\cdots \rightarrow X_{n+1} \stackrel{d_{n+1}}{\rightarrow} X_{n} \stackrel{d_{n}}{\rightarrow} X_{n-1} \rightarrow \cdots$ in $\mathcal{T}$ such that for all integers $n$, there exist $\mathcal{T}(-, \mathcal{P}(\xi))$-exact triangles $K_{n+1} \stackrel{g_{n}}{\rightarrow} X_{n} \stackrel{f_{n}}{\rightarrow}$ $K_{n} \stackrel{h_{n}}{\rightarrow} \Sigma K_{n+1}$ in $\xi$, where the differential $d_{n}$ is defined as $d_{n}=g_{n-1} f_{n}$ for any integer $n$.

A complete $\xi$-projective resolution is a complete $\xi$-exact complex

$$
P: \cdots \longrightarrow P_{n+1} \stackrel{d_{n+1}}{\longrightarrow} P_{n} \stackrel{d_{n}}{\longrightarrow} P_{n-1} \longrightarrow \cdots
$$

in $\mathcal{T}$ such that $P_{n} \in \mathcal{P}(\xi)$ for any integer $n$.

Let $P$ be a complete $\xi$-projective resolution in $\mathcal{T}$. So for any integer $n$, there exists a triangle $K_{n+1} \stackrel{g_{n}}{\rightarrow} X_{n} \stackrel{f_{n}}{\rightarrow} K_{n} \stackrel{h_{n}}{\rightarrow} \Sigma K_{n+1}$ in $\xi$. The object $K_{n}$ is called $\xi$-Gprojective for any integer $n$. We denote by $\mathcal{G} \mathcal{P}(\xi)$ the full subcategory of $\xi-\mathcal{G}$ projective objects of $\mathcal{T}$.

For an object $\mathrm{T}$ of $\mathcal{T}$, we define inductively the $\xi-\mathcal{G}$ projective dimension, $\xi-\mathcal{G} \mathrm{pd} T$. When $T=0$, put $\xi-\mathcal{G} \operatorname{pd} T=-1$. If $T \in \mathcal{G P}(\xi)$, then we define $\xi-\mathcal{G} \operatorname{pd} T=0$. Let $n>0$. We define $\xi-\mathcal{G}$ pd $T=n$ if $\xi-\mathcal{G}$ pd $T \not \leq n-1$ and there exists a triangle $K \rightarrow G \rightarrow A \rightarrow$ $\Sigma K$ in $\xi$ such that $G \in \mathcal{G P}(\xi)$ and $\xi-\mathcal{G}$ pd $K \leqslant n-1$. Finally, if $\xi-\mathcal{G}$ pd $T \neq n$ for all $n \geqslant 0$, we set $\xi-\mathcal{G} \operatorname{pd} T=\infty$. We define $\xi-\mathcal{G P}$-gldim $\mathcal{T}$ as the supremum of $\xi-\mathcal{G} \operatorname{pd} T$ over all $T \in \mathcal{T}$.

Similar (or rather dual) to the way that we define $\xi$-G projective objects, $\xi$ $\mathcal{G}$ projective dimension and $\xi-\mathcal{G} \mathcal{P}$-gldim $\mathcal{T}$, one can define $\xi$-Ginjective objects, $\xi$ $\mathcal{G}$ injective dimension of $\mathcal{T}$ and $\xi-\mathcal{G} \mathcal{I}$-gldim $\mathcal{T}$.

DeFINITION 5.2. We will say that $\mathcal{T}$ is a Gorenstein triangulated category with respect to $\xi$ if for any object $L$ of $\mathcal{T}, \xi-\operatorname{pd} L<\infty$ if and only if $\xi-\mathrm{id} L<\infty$. When we say that $(\mathcal{T}, \mathcal{L})$ is a Gorenstein triangulated category with respect to $\xi$, we mean that $\mathcal{T}$ is such a category and that $\mathcal{L}=\{L \in \mathcal{T} \mid \xi-\operatorname{pd} L<\infty\}$.

Lemma 5.3. Let $X$ be an object of $\mathcal{T}$ with $\xi-\mathcal{G} p d T<\infty$. Then there exists a triangle $X \rightarrow L \rightarrow G \rightarrow \Sigma X$ in $\xi$ such that $G \in \mathcal{G} \mathcal{P}(\xi)$ and $\xi-p d L=\xi-\mathcal{G} p d X$.

Proof. If $X \in \mathcal{G P}(\xi)$, we take $X \rightarrow L \rightarrow G \rightarrow \Sigma X$ to be the first triangle in the 'right half' of a complete $\xi$-projective resolution of $X$. We may assume that $\xi-\mathcal{G} \operatorname{pd} X=$ $n>0$. Then $[1,4.6]$ yields a triangle $K \rightarrow G^{\prime} \rightarrow X \rightarrow \Sigma K$ in $\xi$ such that $G^{\prime} \in \mathcal{G P}(\xi)$ and $\xi-\operatorname{pd} K=n-1$. Since $G^{\prime} \in \mathcal{G P}(\xi)$, there is a triangle $G^{\prime} \rightarrow P \rightarrow G \rightarrow \Sigma G^{\prime}$ in $\xi$ with $P \in \mathcal{P}(\xi)$ and $G \in \mathcal{G P}(\xi)$. Applying cobase change for the triangle $\Sigma^{-1} G \rightarrow$ $G^{\prime} \rightarrow P \rightarrow G$ along $G^{\prime} \rightarrow X$, we have the following commutative diagram: 


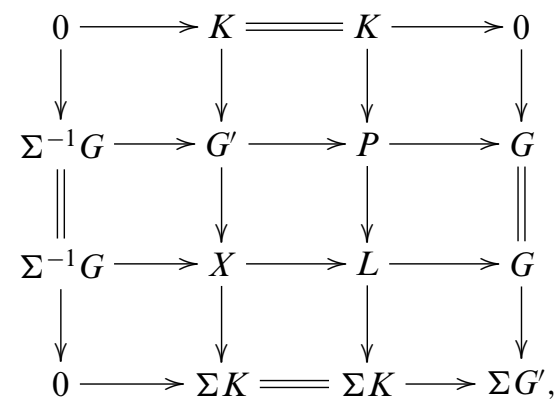

in which the triangle $X \rightarrow L \rightarrow G \rightarrow \Sigma X$ is in $\xi$. We now argue that $\xi$-pd $L=n$. If $L \in \mathcal{P}(\xi)$, then $\xi-\mathcal{G} \operatorname{pd} X=0$ by $[\mathbf{1}, 3.11]$, which is a contradiction. Thus, $\xi-\operatorname{pd} L>0$. If $\xi-\operatorname{pd} L \leqslant n-1$, then $\xi-\mathcal{G} \operatorname{pd} X \leqslant n-1$ by $[1,3.14]$. It follows that $\xi-\operatorname{pd} L=\xi-\mathcal{G} \operatorname{pd} X$.

LEMMA 5.4. Let $(\mathcal{T}, \mathcal{L})$ be a Gorenstein triangulated category with $\xi-\mathcal{G P}$-gldim $\mathcal{T} \leqslant n$ for some non-negative integer $n$. Then $\left({ }^{\perp} \mathcal{L}, \mathcal{L}\right)$ is a complete cotorsion pair with respect to $\xi$ and ${ }^{\perp} \mathcal{L}=\{T \in \mathcal{T} \mid \xi(T, L)=0$ for all $L \in \mathcal{L}\}=\mathcal{G P}(\xi)$.

Proof. First we show that ${ }^{\perp} \mathcal{L}$ is the class of $\xi$-G projective objects of $\mathcal{T}$. Let $X \in$ ${ }^{\perp} \mathcal{L}$. Lemma 5.3 gives us a triangle $X \rightarrow L \rightarrow G \rightarrow \Sigma X$ in $\xi$ such that $G \in \mathcal{G P}(\xi)$ and $\xi-\operatorname{pd} L=\xi-\mathcal{G} \operatorname{pd} X \leqslant n$. For $L$, there exists a triangle $L^{\prime} \rightarrow P \rightarrow L \rightarrow \Sigma L^{\prime}$ in $\xi$ with $P \in \mathcal{P}(\xi)$ and $\xi-\operatorname{pd} L^{\prime} \leqslant n-1$. Applying base change for the triangle $L^{\prime} \rightarrow P \rightarrow L \rightarrow$ $\Sigma L^{\prime}$ along $X \rightarrow L$, we have the following commutative diagram:

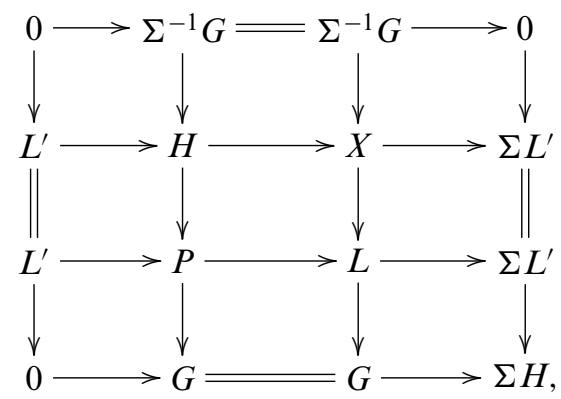

in which the second horizontal triangle is in $\xi$. Hence, Proposition 2.3 implies that $H \rightarrow P \rightarrow G \rightarrow \Sigma H$ is in $\xi$. Note that $L^{\prime} \in \mathcal{L}$, so the second horizontal triangle in the above diagram is split and $X \rightarrow P$ is a $\xi$-proper monic. Hence, there exists a commutative diagram:

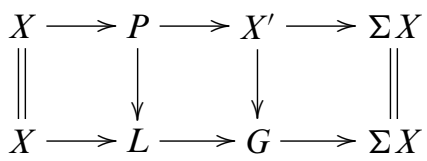

with rows in $\xi$. But the second row is $\mathcal{T}(-, \mathcal{P}(\xi))$-exact, so is the first row. It follows that $X^{\prime} \in{ }^{\perp} \mathcal{L}$. By repeating this process, we obtain a $\mathcal{T}(-, \mathcal{P}(\xi))$-exact $\xi$-exact sequence $0 \rightarrow$ $X \rightarrow P_{0} \rightarrow P_{-1} \rightarrow \cdots$ with each $P_{i} \in \mathcal{P}(\xi)$. Thus, [1, 3.19] shows that $X \in \mathcal{G P}(\xi)$.

Next, we show that $\left({ }^{\perp} \mathcal{L}, \mathcal{L}\right)$ is complete with respect to $\xi$. By $[1,4.6],\left({ }^{\perp} \mathcal{L}, \mathcal{L}\right)$ has enough $\xi$-projectives. Let $T \in \mathcal{T}$ and consider the triangle $T \rightarrow I \rightarrow C \rightarrow \Sigma T$ in $\xi$ with $I \in \mathcal{I}(\xi)$. For $C$, there is a triangle $K \rightarrow G \rightarrow C \rightarrow \Sigma K$ in $\xi$ with $G \in \mathcal{G P}(\xi)$ and 
$K \in \mathcal{L}$. Applying base change for the triangle $T \rightarrow I \rightarrow C \rightarrow \Sigma T$ along $G \rightarrow C$, we have the following commutative diagram:

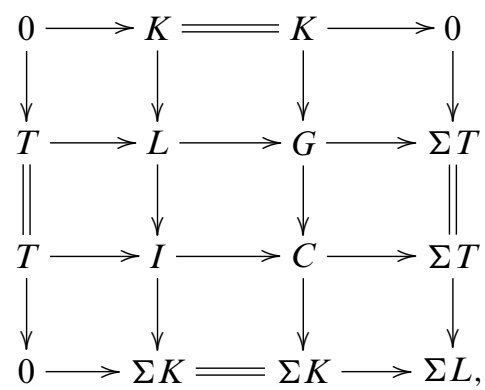

in which the second horizontal and the second vertical triangles are in $\xi$. But $K, I \in \mathcal{L}$, then $L \in \mathcal{L}$. The second horizontal triangle shows that $\left({ }^{\perp} \mathcal{L}, \mathcal{L}\right)$ has enough $\xi$-injectives. This completes the proof.

By analogy with the proof of Lemmas 5.3 and 5.4, we have the following results.

Lemma 5.5. Let $X$ be an object of $\mathcal{T}$ with $\xi-\mathcal{G}$ idT $<\infty$. Then there exists a triangle $G \rightarrow L \rightarrow X \rightarrow \Sigma G$ in $\xi$ such that $G \in \mathcal{G I}(\xi)$ and $\xi-i d L=\xi-\mathcal{G} i d X$.

LEMMA 5.6. Let $(\mathcal{T}, \mathcal{L})$ be a Gorenstein triangulated category with $\xi-\mathcal{G} \mathcal{I}$-gldim $\mathcal{T} \leqslant n$ for some non-negative integer $n$. Then $\left(\mathcal{L}, \mathcal{L}^{\perp}\right)$ is a complete cotorsion pair with respect to $\xi$, and $\mathcal{L}^{\perp}=\{T \in \mathcal{T} \mid \xi(L, T)=0$ for all $L \in \mathcal{L}\}=\mathcal{G} \mathcal{I}(\xi)$.

By the above arguments and [1, 3.17], we get the following assertion.

THEOREM 5.7. Let $(\mathcal{T}, \mathcal{L})$ be a Gorenstein triangulated category with $\xi-\mathcal{G P}$-gldim $\mathcal{T} \leqslant n$ and $\xi-\mathcal{G} \mathcal{I}$-gldim $\mathcal{T} \leqslant n$ for some non-negative integer $n$. Then there are two triangulated model structures with respect to $\xi$ on $\mathcal{T}$, where the class of trivial objects is $\mathcal{L}$. In the projective triangulated model structure, every object is fibrant, and the cofibrant objects are the $\xi$-Gprojective objects. In the injective triangulated model structure, every object is cofibrant, and the fibrant objects are the $\xi$-Ginjective objects.

ACKNOWLEDGEMENTS. This research was partially supported by the Program for New Century Excellent Talents in University (NCET-13-0957) and NSFC (11361051 and 11361052). The author would like to thank the referees for helpful suggestions and corrections.

\section{REFERENCES}

1. J. Asadollahi and Sh. Salarian, Gorenstein objects in triangulated categories, J. Algebra 281 (2004), 264-286.

2. A. A. Beilinson, J. Bernstein and P. Deligne, Perverse sheaves, in Analysis and topology on singular spaces I. (Luminy, 1981); Astérisque 100 (1982), 5-171.

3. A. Beligiannis, Relative homological algebra and purity in triangulated categories, $J$. Algebra 227 (2000), 268-361.

4. T. Bühler, Exact categories, Expo. Math. 28 (2010), 1-69.

5. J. Gillespie, Model structures on exact categories, J. Pure Appl. Algebra 215 (2011), 2892-2902. 
6. R. Hartshorne, Residues and duality, Lecture notes of a seminar on the work of A. Grothendieck given at Harvard 1963/64, Lecture Notes in Math., vol. 20 (Springer-Verlag, Berlin, Germany, 1966.

7. M. Hovey, Model categories (American Mathematical Society, Providence, RI, 1999.

8. M. Hovey, Cotorsion pairs, model category structures, and representation theory, Math. Z. 241 (2002), 553-592.

9. A. Hubery, Notes on the octahedral axiom. Available at http://www.maths. leeds.ac.uk/ahubery/octahedral.pdf.

10. B. Iversen, Octahedra and braids, Bull. Soc. Math. France 114 (1986), 197-213.

11. D. Murfet, Triangulated categories part I, April 11, 2007. Available at Therisingsea.org/notes/TriangulatedCategories.pdf.

12. A. Neeman, Triangulated categories, Ann. Math. Stud., vol. 148, (Princeton University Press, 2001).

13. D. Puppe, On the structure of stable homotopy theory, Colloquium on algebraic topology (Aarhus Universitet Matematisk Institut 1962) 65-71. 